\title{
Detection of Postictal Generalized Electroencephalogram Suppression: Random Forest Approach
}

Xiaojin $\mathrm{Li}^{1}$, PhD; Shiqiang Tao ${ }^{1}$, PhD; Shirin Jamal-Omidi ${ }^{1}$, MD; Yan Huang ${ }^{2}$, MSc; Samden D Lhatoo ${ }^{1}$, MD; Guo-Qiang Zhang ${ }^{1,3}$, PhD; Licong $\mathrm{Cui}^{3}$, $\mathrm{PhD}$

${ }_{1}^{1}$ Department of Neurology, University of Texas Health Science Center, Houston, TX, United States
${ }^{2}$ Department of Computer Science, University of Kentucky, Lexington, KY, United States
${ }^{3}$ School of Biomedical Informatics, University of Texas Health Science Center, Houston, TX, United States

Corresponding Author:

Licong Cui, PhD

School of Biomedical Informatics

University of Texas Health Science Center

7000 Fannin St

Houston, TX, 77030

United States

Phone: 17135003791

Email: licong.cui@uth.tmc.edu

\section{Abstract}

Background: Sudden unexpected death in epilepsy (SUDEP) is second only to stroke in neurological events resulting in years of potential life lost. Postictal generalized electroencephalogram (EEG) suppression (PGES) is a period of suppressed brain activity often occurring after generalized tonic-clonic seizure, a most significant risk factor for SUDEP. Therefore, PGES has been considered as a potential biomarker for SUDEP risk. Automatic PGES detection tools can address the limitations of labor-intensive, and sometimes inconsistent, visual analysis. A successful approach to automatic PGES detection must overcome computational challenges involved in the detection of subtle amplitude changes in EEG recordings, which may contain physiological and acquisition artifacts.

Objective: This study aimed to present a random forest approach for automatic PGES detection using multichannel human EEG recordings acquired in epilepsy monitoring units.

Methods: We used a combination of temporal, frequency, wavelet, and interchannel correlation features derived from EEG signals to train a random forest classifier. We also constructed and applied confidence-based correction rules based on PGES state changes. Motivated by practical utility, we introduced a new, time distance-based evaluation method for assessing the performance of PGES detection algorithms.

Results: The time distance-based evaluation showed that our approach achieved a 5-second tolerance-based positive prediction rate of 0.95 for artifact-free signals. For signals with different artifact levels, our prediction rates varied from 0.68 to 0.81 .

Conclusions: We introduced a feature-based, random forest approach for automatic PGES detection using multichannel EEG recordings. Our approach achieved increasingly better time distance-based performance with reduced signal artifact levels. Further study is needed for PGES detection algorithms to perform well irrespective of the levels of signal artifacts.

(JMIR Med Inform 2020;8(2):e17061) doi: 10.2196/17061

\section{KEYWORDS}

epilepsy; generalized tonic-clonic seizure; postictal generalized EEG suppression; EEG; random forest

\section{Introduction}

\section{Background}

Epilepsy is one of the most common neurological disorders, and it affects an estimated 65 million people worldwide [1]. An epileptic seizure (hereafter referred to as seizure) is a brief episode, usually with signs or symptoms because of transient, undesired, excessive, and synchronous electrical discharge, involving large numbers of neurons in the brain [2]. When seizure occurs, altered movement, expression, and levels of consciousness are often observed in the affected person. Seizure 
may produce temporary confusion, uncontrollable jerking movements of the arms and legs, inability to speak, or loss of consciousness or awareness [3].

In a worst-case scenario, frequent seizures may predispose a person to sudden unexpected death in epilepsy (SUDEP) [4]. Among neurological events and conditions, SUDEP is second only to stroke in years of potential life lost, highlighting the importance and significance of this condition for public health [5]. SUDEP is a catastrophic and fatal complication of epilepsy. The definition of SUDEP is "sudden, unexpected, witnessed or unwitnessed, non-traumatic and non-drowning death, occurring in benign circumstances, in an individual with epilepsy, with or without evidence for a seizure and excluding documented status epilepticus, in which postmortem examination does not reveal a cause of death" [6], that is, no other cause of death can be found [7]. However, the mechanisms underlying SUDEP are not completely understood.

Electrophysiological signals such as electroencephalogram (EEG), electrocardiogram, and electromyography, collected together in the epilepsy monitoring unit (EMU), are traditionally used for understanding epileptic seizures [8]. Noninvasive scalp EEG and invasive intracranial EEG are the most commonly used methods for locating seizures and monitoring the interphase activity between seizures [9]. Invasive intracranial EEG is one of the techniques used in localizing the seizure onset zone in preparation for surgery [8]. EEG is a key source of information for the diagnosis of epilepsy, including whether epilepsy is focal or generalized, idiopathic or symptomatic, or part of a specific epilepsy syndrome [10]. Therefore, EEG has also been widely used to identify biomarkers that can help prevent the development of epilepsy, identify focal brain regions that produce epilepsy, and ultimately cure epilepsy through surgical means [11].

Postictal generalized EEG suppression (PGES) is a potential EEG biomarker of SUDEP risk [12-14]. PGES is a period of brain inactivity after seizure. It most often occurs after generalized tonic-clonic seizures (GTCS), particularly in those arising from sleep, and is related to the symmetric tonic phase, postictal immobility, lack of early oxygen administration, duration of oxygen desaturation, and lower peripheral capillary oxygen saturation nadir values [15-17]. GTCS are the most significant risk factor for SUDEP [13]. PGES is defined as a diffused EEG background attenuation $(<10 \mu \mathrm{V})$ in the postictal period [18]. Prolonged PGES ( $>50$ seconds) has been reported in refractory epilepsy patients who are at risk of SUDEP [14]. For every prolonged second in the duration of PGES, the odds of SUDEP is increased by a factor of $1.7 \%(P<.005)$ [14]

Clinically, the determination of the duration of PGES is manually performed by human experts through visual inspection of EEG signals. According to definition, the identification of PGES appears to be straightforward by identifying a period of low-amplitude EEG signals after the seizure, as shown in Figure 1. However, real-world data recorded in EMUs may contain high-amplitude signals caused by physiological artifacts (eg, breathing, muscle, and movement artifacts), as shown in Figure 2. Therefore, clinical experts usually leverage additional video recordings along with signals to identify high-amplitude artifacts that are not real EEG activities [19]. Automated PGES detection tools are highly desirable to assist clinical personnel in reviewing and annotating PGES in EEG recordings. Automated techniques have been extensively studied for epilepsy-related EEG signal analysis [20], including a random forest classifier with empirical wavelet transform for seizure identification [21], a data-driven approach for classifying seizure and nonseizure EEG signals using the multivariate empirical mode decomposition algorithm [22], a whole-brain seizure detection approach using the K-nearest neighbors classifier [23], and extreme epileptic events detection and prediction using neural networks with time-frequency features [24,25]. However, there has been only one study [19] using logistic regression to perform automated PGES detection based on frequency-domain features of EEG signals. The following challenges remain in developing a fully automatic PGES detection tool:

- The presence of artifacts remains the main challenge that makes PGES detection more complex than applying a fixed amplitude threshold.

- There is no sensitive and standardized criterion dedicated to measuring and evaluating the performance of PGES detection algorithms.

Figure 1. An example of postictal generalized electroencephalogram suppression and intermittent slow wave activity signals after generalized tonic-clonic seizures. GTCS: generalized tonic-clonic seizures; ISW: intermittent slow wave; PGES: postictal generalized electroencephalogram suppression.

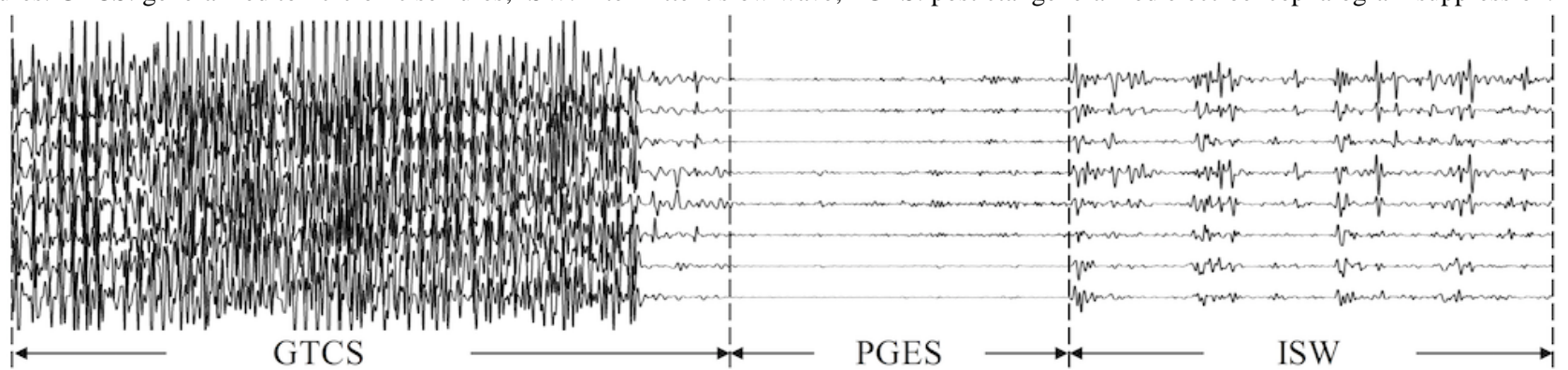


Figure 2. An example of postictal generalized electroencephalogram suppression and intermittent slow wave activity signals (with artifacts) after generalized tonic-clonic seizures. GTCS: generalized tonic-clonic seizures; ISW: intermittent slow wave; PGES: postictal generalized electroencephalogram suppression.

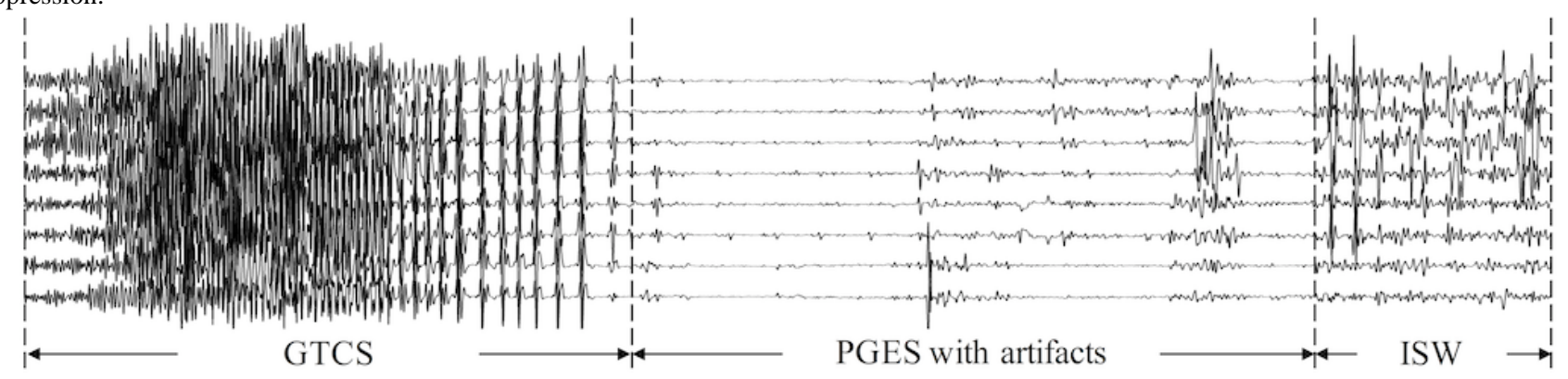

In this paper, we introduce a random forest-based classifier for PGES detection by leveraging a variety of EEG signal features such as time-domain features, frequency-domain features, wavelet-based features, and interchannel correlations. We incorporate confidence-based correction rules to remove suspicious sudden changes of EEG activities. This study focused on identifying the first slow wave brain activity, that is, the onset of the first intermittent slow wave (ISW) activity (see Figure 1 and Figure 2), which indicates that the brain activity will gradually recover [12,14]. Therefore, the output of our PGES detection method for each signal recording is the onset of the first ISW. Accordingly, traditional segment-based performance evaluation methods are not well suited for PGES detection. Instead, we introduced a new, recording-by-recoding evaluation method dedicated to PGES detection with direct practical relevance.

\section{The Center for Sudden Unexpected Death in Epilepsy Research}

The Center for SUDEP Research (CSR) is a National Institute of Neurological Disorders and Stroke-funded Center Without Walls initiative for collaborative research on epilepsy. It comprises researchers from 14 institutions across the United States and Europe, bringing together extensive and diverse expertise to understand SUDEP $[4,26]$. The goal of CSR is to better understand cortical, subcortical, and brainstem mechanisms responsible for SUDEP and to use a data-driven, systems biology approach to elucidate the role of cortical influences in SUDEP. To advance SUDEP research, CSR created an infrastructure to fully, effectively, and efficiently utilize a range of prospectively collected data from different domains, including clinical, electrophysiological, biochemical, genetic, and neuropathological fields. CSR provides a comprehensive, curated repository of prospectively collected multimodal data, including electrophysiological signals in European data format. These data are linked to risk factor and outcomes data of over 2500 epilepsy patients (a broad spectrum of ages as well as social, racial, and ethnic groups) with thousands of 24-hour recordings.

\section{Feature Extraction From Electroencephalogram Signals}

For EEG signal feature extraction, the following 4 categories of features are considered in this work: (1) time-domain features,

(2) frequency-domain features, (3) wavelet-based features, and (4) interchannel correlations.
1. Time-domain features: Time-domain features include statistical measures and Hjorth parameters. Statistical measures include nth percentile of the signal, average, range, standard deviation, skewness, and kurtosis [27]. Here, the mean measures the central tendency, skewness measures the asymmetry, and kurtosis measures the tailedness of a probability distribution. Hjorth parameters are commonly used for feature extraction to perform EEG signal analysis [28], including mobility and complexity [29-31]. The mobility represents the mean frequency or the proportion of the standard deviation of the power spectrum. The complexity indicates the signal's similarity to a pure sine wave [31].

2. Frequency-domain features: An EEG wave (captured by an electrode) comprises many other waves with different amplitudes and frequencies. Therefore, an EEG signal has different bands, defined by the frequency of the waves, such as slow oscillations $(0.5 \mathrm{~Hz}-1 \mathrm{~Hz})$, delta bands $(1 \mathrm{~Hz}-4$ $\mathrm{Hz}$ ), theta bands $(4 \mathrm{~Hz}-8 \mathrm{~Hz})$, alpha bands $(8 \mathrm{~Hz}-12 \mathrm{~Hz})$, beta bands $(14 \mathrm{~Hz}-30 \mathrm{~Hz})$, and gamma bands $(30 \mathrm{~Hz}-80$ $\mathrm{Hz}$ ). The spectral power in a specific frequency band, for instance, the $0.5 \mathrm{~Hz}$ to $1 \mathrm{~Hz}$ band, can be regarded as a feature.

3. Wavelet-based features: Wavelets are a relatively recent approach for signal processing, and the main advantage is that wavelets allow multiresolution analysis in time and frequency simultaneously [32,33].

4. Interchannel correlations: Many studies have attempted to find movement-related information of connectivity between different brain regions [34-38]. Correlation analysis represents the degree of relatedness and synchrony between 2 time series. It indicates similar information as a cross-coherence analysis of different EEG channels [39].

\section{Random Forest}

Random forest is an ensemble learning method used for classification and regression problems. This method has been used for automated sleep stage classification based on EEG signals [40,41]. Random forest involves a group of decision trees during training and outputs the mode of the classes predicted by individual trees. The overall output is determined by applying the input to each tree and choosing the class that gets the most weighted vote. The weight of each tree is adjusted using misclassification and out-of-bag measures.

As it is different from other traditional classifiers (eg, K-nearest neighbor, support vector machine, and artificial neural network), 
we select random forest as the approach for our study because of the following advantages: (1) adaptable, as it estimates the importance of variables and provides a way for tuning with additional training data by assigning different weights for each decision tree; (2) scalable, as it can handle thousands of input variables and work efficiently on large datasets; and (3) robust, as it can balance errors in datasets with unbalanced class population [41].

\section{Methods}

\section{Overview}

The dataset used for this study comprises 116 EEG signal recordings from 84 patients with GTCS in the CSR data repository, with PGES annotated by domain experts. We extracted the 5-min postictal EEG signals for PGES detection. A total of 8 EEG channels are utilized: Fp1-F7, F7-T7, T7-P7, Fp2-F8, F8-T8, T8-P8, Fz-Cz, and Cz-Pz.

The overall workflow of our PGES detection method is shown in Figure 3. The process started with the preprocessing of the EEG signals (step 1), followed by feature extraction (step 2). Then a random forest classifier was trained and tested based on the extracted features (step 3). We applied correction rules, which are constructed based on the continuity of brain activities, to the prediction of the random forest (step 4) and provided the final detected label for each signal segment (step 5).

Figure 3. The overall workflow of our automated postictal generalized electroencephalogram suppression detection approach. EEG: electroencephalogram; PGES: postictal generalized electroencephalogram suppression.

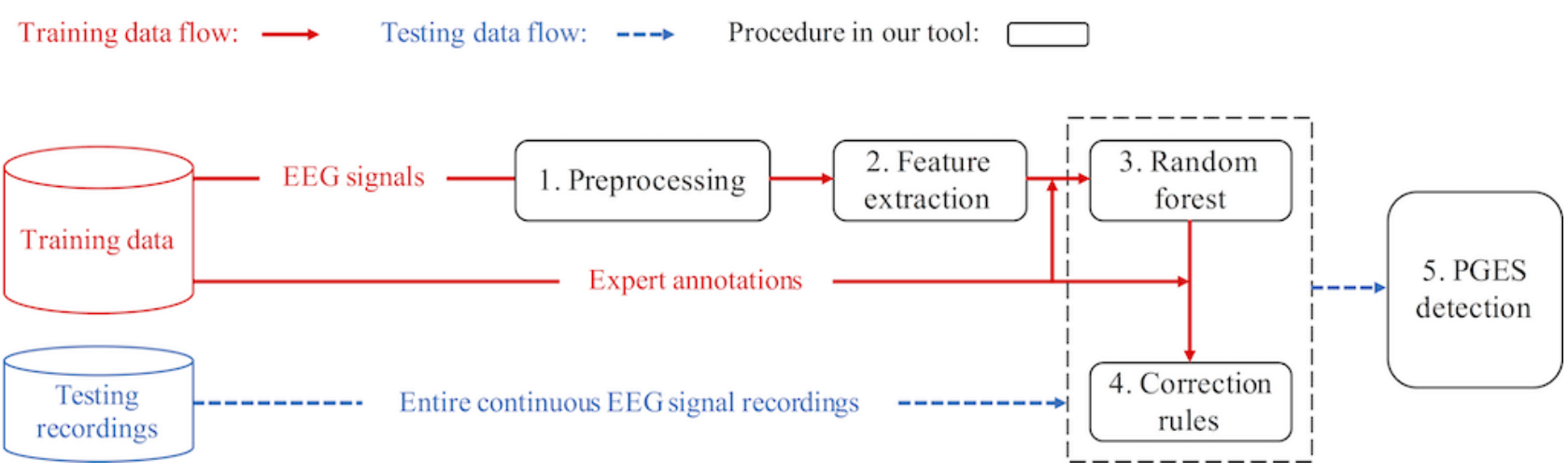

\section{Preprocessing}

Each postictal EEG signal record is split into signal segments with a length of 1 second (ie, 1-second epoch) from the beginning to the end without overlapping. The common electrophysiological artifacts present in the EEG signal recordings include muscle artifacts, breathing, and body and bed movements [42]. The main frequency of ISW is less than $5 \mathrm{~Hz}$. To minimize the presence of residual artifacts, the signals are filtered with a band-pass filter with cutoff frequencies at 0.5 $\mathrm{Hz}$ and $5 \mathrm{~Hz}$.

\section{Feature Extraction}

For each signal segment of the 8 EEG channels, we extracted 76 features including time-domain features, frequency-domain features, and wavelet-based features as follows:

- The following 16 time-domain features were extracted: (1) 11 statistic features, including mean, median, maximum, minimum, range, standard deviation, $n$th percentile of the signal ( $n=5,25,75$, and 95), and the root mean square, and (2) 5 time-domain properties of kurtosis, skewness, mobility, complexity, and amplitude energy (AE) of the signal. The time-domain properties for a time series $X=\left\{x_{l}\right.$, $\left.x_{2}, \ldots, x_{n}\right\}$ are defined in Figure 4 [27-30], where $N$ is the number of data points, $\bar{x}$ is the mean of $X$, and $d_{i}=x_{i}-x_{i}-{ }_{1}$ and $i=1, \ldots, n$.

- A total of 4 frequency-domain features were extracted. As the PGES and ISW are typically low-frequency brain activities $(0.5 \mathrm{~Hz}-5 \mathrm{~Hz})$, we extracted the spectral power of 4 low-frequency bands consisting of $0.5 \mathrm{~Hz}$ to $1 \mathrm{~Hz}, 1$ $\mathrm{Hz}$ to $2 \mathrm{~Hz}, 2 \mathrm{~Hz}$ to $4 \mathrm{~Hz}$, and $4 \mathrm{~Hz}$ to $5 \mathrm{~Hz}$.

- A total of 56 wavelet-based features were extracted. EEG signals were subjected to three-level decomposition using the Daubechies 4 wavelet. From the decomposition process, a total of 4 coefficient sets were generated, and we calculated 14 measurements (except range and AE) used in time-domain features for each coefficient set as wavelet-based features [32,33]. 
Figure 4. The definitions of the time-domain properties. AE: amplitude energy.

$$
\begin{aligned}
& \text { Kurtosis }=\frac{\frac{1}{N} \sum_{i=1}^{N}\left(x_{i}-\bar{x}\right)^{4}}{\left[\frac{1}{N-1} \sum_{i=1}^{N}\left(x_{i}-\bar{x}\right)^{2}\right]^{2}}-3(\mathrm{a}) \\
& \text { Skewness }=\frac{\frac{1}{N} \sum_{i=1}^{N}\left(x_{i}-\bar{x}\right)^{3}}{\left[\frac{1}{N-1} \sum_{i=1}^{N}\left(x_{i}-\bar{x}\right)^{2}\right]^{3 / 2}}(\mathrm{~b}) \\
& \text { Mobility }=\sqrt{\frac{\sum_{i=1}^{N} d_{i}}{\sum_{i=1}^{N} x_{i}}(\mathrm{c})} \\
& \text { Complexity }=\sqrt{\frac{\left[\sum_{i=1}^{N}\left(d_{i}-d_{i-1}\right)^{2}\right] \times \sum_{i=1}^{N} x_{i}}{\left(\sum_{i=1}^{N} d_{i}\right)^{2}}}(\mathrm{~d}) \\
& \text { AE }=\sum_{i=1}^{N}\left|x_{i}\right|(\mathrm{e})
\end{aligned}
$$

To capture cross-coherence of EEG channels, we further investigated the interchannel correlations using the linear correlation coefficient between selected channels. The correlation coefficient for 2 time series, $X=\left\{x_{1}, x_{2}, \ldots, x_{n}\right\}$ and $Y=\left\{y_{1}, y_{2}, \ldots, y_{n}\right\}$ is defined in Figure 5 [39], where $N$ is the number of data points and $\bar{x}$ is the mean. For each signal segment, we calculated 4 interchannel correlations: $\operatorname{corr}(F p 1-F 7, F p 2-F 8), \operatorname{corr}(F p 2-F 8, F z-C z), \operatorname{corr}(F p 1-F 7$, $F z-C z)$, and $\operatorname{corr}(F z-C z, C z-P z)$.

Figure 5. The definitions of the linear correlation coefficient between two time series ( $\mathrm{X}$ and $\mathrm{Y}$ ).

$$
\operatorname{corr}(X, Y)=\frac{\sum_{i=1}^{N}\left(x_{i}-\bar{x}\right) \times\left(y_{i}-\bar{y}\right)}{\sqrt{\sum_{i=1}^{N}\left(x_{i}-\bar{x}\right)^{2}} \sqrt{\sum_{i=1}^{N}\left(y_{i}-\bar{y}\right)^{2}}}
$$

\section{Random Forest Classifier}

There are 5 steps to build the random forest classifier with the bootstrap aggregating (bagging) technique [43], which is an ensemble method to reduce the variance without increasing the bias for decision tree algorithms. Given a training set $X=\left\{x_{1}, x_{2}\right.$ $\left., \ldots, x_{n}\right\}$ and $Y=\left\{y_{1}, y_{2}, \ldots, y_{n}\right\}$, the 5 steps are as follows:

1. Generate a subtraining set $S=\left\{X_{s}, Y_{s}\right\}$ by selecting a random number of observations and features from the whole input training dataset.

2. Build and train each decision tree $T_{i}$ (eg, regression tree, random tree, and $\mathrm{C} 4.5$ ) with the generated subtraining set $S$. In the construction of each decision tree, nodes and leaves are built by selecting a random number of features. This process will minimize the correlation among the features and decrease the sensitivity to noise [40].

3. Estimate out-of-bag errors. In the training process of each tree, about two-thirds of $S$ are used for tree construction, and the remaining one-third is used to test the classification performance of the tree. Therefore, it gets an unbiased estimate of the test set error internally in a random forest, and there is no need to use further cross-validation [43].

4. Repeat the above steps ( $\operatorname{step} 1$, step 2, and step 3) $N$ times to build $N$ decision trees $T=\left\{T_{1}, T_{2}, \ldots, T_{N}\right\}$.
5. Compute the classifier output. After training, the output $y^{\prime}$ for an unknown sample $x^{\prime}$ can be made by averaging the output from all the individual decision trees on $x^{\prime}$ :

$$
\frac{1}{N} \sum_{i=1}^{N} T_{i}\left(x^{\prime}\right)
$$

This classifier can obtain strongly correlated trees by training all trees with the same training set, and bagging is a way to decorrelate the trees. The prediction results of a single decision tree may be highly sensitive to noises in its particular training set, especially with overfitting. However, in a random forest, the average of all trees is less sensitive to noises as the trees are more decorrelated. In this work, we used a random forest with $N=1000$ trees.

\section{Correction Rules for Continuous Detection}

According to the knowledge of the domain experts, longtime EEG suppression does not often occur after the first ISW happens in practical scenarios. This indicates that sudden changes of PGES/ISW states are unlikely to happen. For example, for a sequence of predicted labels with 10 consecutive 1-second segments (PGES, PGES, PGES, ISW, PGES, PGES, PGES, PGES, PGES, and PGES), the sudden changes from PGES to ISW (from the third segment to the fourth segment) and from ISW back to PGES (from the fourth segment to the fifth segment) are unlikely, that is, the predicted label for the fourth segment is most likely a misclassification and should be 
corrected and replaced with PGES. Therefore, we considered the temporal contextual information of segments to perform correction.

We constructed confidence-based correction rules based on the probability output of the random forest classifier. For each segment $\operatorname{Seg}_{i}$, we built a confidence index, $\operatorname{conf}\left(\operatorname{Seg}_{i}\right)$ based on the average probability from the current segment to the next $M$ segments; the definition is as follows:

$$
\operatorname{conf}\left(\operatorname{Seg}_{i}\right)=\frac{1}{M+1} \sum_{j=i}^{i+M} \operatorname{prob}\left(\operatorname{Seg}_{j}\right)
$$

where $\operatorname{prob}\left(\mathrm{Seg}_{j}\right)$ is the random forest probability output of the segment $\mathrm{Seg}_{j}$.

In this work, we chose $M=5$. On the basis of the probability and confidence index of each segment, we applied the following 3 rules to correct suspicious sudden changes of PGES/ISW states in the detected label sequence:

1. If $\operatorname{prob}\left(\mathrm{Seg}_{i}\right)$ had a high value but the probabilities of $\mathrm{Seg}_{i}$ 's surrounding segments (eg, $\operatorname{prob}\left(\operatorname{Seg}_{i-1}\right)$ and $\operatorname{prob}\left(\operatorname{Seg}_{i+1}\right)$ had low values, then we corrected the detected label of Segi as PGES.

2. If $\operatorname{conf}\left(\operatorname{Seg}_{i}\right)$ had a high value but the confidence indexes of $\mathrm{Seg}_{i}$ 's surrounding segments (eg, $\operatorname{conf}\left(\operatorname{Seg}_{i-1}\right)$ and $\left.\operatorname{conf}\left(\operatorname{Seg}_{i+1}\right)\right)$ had low values, then we corrected the detected label of $\mathrm{Seg}_{i}$ as PGES.

3. If $\operatorname{prob}\left(\mathrm{Seg}_{i}\right)$ had a high value but $\operatorname{conf}\left(\operatorname{Seg}_{i}\right)$ had a low value, then we corrected the detected label of $\mathrm{Seg}_{i}$ as PGES.

\section{Performance Evaluation}

For traditional EEG signal classification tasks such as sleep stage classification $[40,44,45]$, the performance evaluations are segment-based (ie, the predictions for each segment determine the performance metrics such as accuracy, precision, and recall). However, for the PGES detection setting, the prediction result of the onset of the first ISW in a given signal recording (ie, recording-based) is more important as it indicates the end of PGES. In other words, the prediction results of segments after the first ISW become less important for the evaluation. Figure 6 shows an example of the signal that is split into 30 segments of 1-second each, and each segment is annotated with a label 0 for PGES or 1 for ISW (see the annotated labels). The predicted labels are generated by the automatic detection method; the predicted label highlighted in bold means that the label is wrongly predicted, whereas other labels are correctly predicted. In this example, only one label is wrongly predicted, indicating that the PGES detection method achieves a high accuracy of 97\% (29/30) for the segment-based evaluation. However, the actual first ISW of the signal is 15 seconds away from the predicted first ISW, a 15 -second time difference that may not be acceptable in clinical scenarios.

Therefore, for the first time, we proposed time distance-based metrics to evaluate an automated PGES detection method. For a given signal recording $r$, we defined the predicted time distance $T D_{r}$ as the time difference between the predicted end time of PGES (or the predicted time of the first ISW) by the detection method and the actual end time of PGES (or the actual time of the first ISW) according to the expert annotations. A lower value of the time distance indicates a better performance of the PGES detection method.

On the basis of the predicted time distance, we further introduced the 5-second tolerance-based positive prediction rate $\left(\mathrm{PPR}_{5 \mathrm{~s}}\right)$ as another evaluation metric, as a 5-second time distance is acceptable according to clinical experts. Given a collection $R$ of signal recordings for evaluation, we define $P P R_{5 s}$ as follows:

$$
P P R_{5 s}=\frac{\left|\left\{r \in R \mid T D_{r} \leq 5 s\right\}\right|}{|R|}
$$

that is, the number of signal recordings whose predicted time distances are within 5 seconds divided by the total number of the signal recordings.

Figure 6. An example of automatic postictal generalized electroencephalogram suppression detection evaluation. Annotated labels are the expert-annotated labels, and predicted labels are generated using the automatic detection algorithm. ${ }^{\mathrm{a}} \mathrm{AL}$ : annotated label; ${ }^{\mathrm{b}} \mathrm{PL}$ : predicted label.

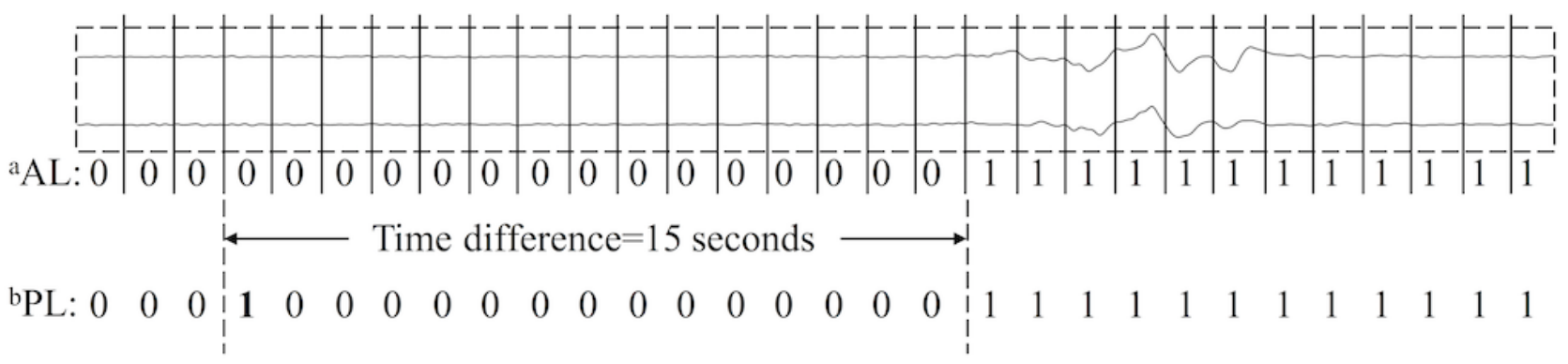

\section{Results}

\section{Artifact Level}

To evaluate the performance of our PGES detection method on EEG signals with different levels of artifacts, we categorized EEG signals into 4 levels: artifact-free, mild artifact, moderate artifact, and severe artifact. The domain expert manually reviewed the EEG signals and classified them into 4 levels according to the following criteria:

1. Artifact-free: No waveforms in any channels or abrupt waveforms of less than a second duration. 
2. Mild: Abrupt waveforms in channels other than midline channels (Fz-Cz and $\mathrm{Cz}-\mathrm{Pz}$ ) that do not affect the midline channels or midline channels involved with abrupt waveforms of less than 1-second duration.

3. Moderate: One of the midline channels is involved, or both midline channels are involved with waveforms of less than 1 -second duration.

4. Severe: Both midline channels have abrupt waveforms of more than 1-second duration. An expert may need to analyze all EEG chains, which include 19 EEG channels, or use video recordings to differentiate the artifacts from the brain-generated waveforms.

Among the 116 signal recordings in our dataset, 27 are artifact-free, 31 are with mild artifacts, 25 are with moderate artifacts, and 33 are with severe artifacts. We applied our PGES detection method to 4 groups of signal recordings with different levels of artifacts: only artifact-free (group A); artifact-free and mild artifact (group B); artifact-free, mild artifact, and moderate artifact (group C); and all signal recordings (group D).

\section{Cross-Validation}

Cross-validation has been generally used for evaluating a model's performance with low bias and variance. We applied 10-fold cross-validation 10 times to the 4 groups with varying artifact levels to evaluate our PGES detection method. For each group, we randomly separated the signal recordings into two parts each time, training set and testing set, and then calculated the evaluation metrics. Note that there was no overlap between the training set and testing set. For instance, in group D, which included all 116 signal recordings, 11 recordings were used as the testing data and the remaining 105 recordings were used as the training data in each fold. We repeated this procedure 10 times and used the average as the final evaluation result.

For the training set, we selected balanced numbers of PGES and ISW segments for each signal recording. Although every EEG recording was annotated by domain experts with the start of the first ISW, there existed EEG recordings missing the annotations of the end of the first ISW (as the onset of the first ISW is the most important). Therefore, for ISW signal segments, we selected up to 30 -second signal (ie, 30 segments) after the onset of the first ISW as follows: if the duration of PGES (say $t$ seconds) is less than 30 seconds, then we used $t$ segments after the first onset of ISW; otherwise, we used 30 segments after the first onset of ISW. For the testing set, we used the entire 5 min of each signal recording to detect the first onset of ISW.

The average predicted time distance is 2.4 seconds for artifact-free signal recordings (group A) and 4.34 seconds for the group containing both artifact-free and mild artifact signal recordings (group B). As the artifact level increases, the average predicted time distance increases as well. The average predicted time distance is 7.54 seconds for the group containing artifact-free, mild artifact, and moderate artifact signal recordings (group C) and 7.84 seconds for all signal recordings (group D).

The $P P R_{5 s}$ of our PGES detection method for each artifact group of signal recordings is as follows: $P P R_{5 s}$ is 0.95 for artifact-free signal recordings (group A); 0.81 for the group containing both artifact-free and mild artifact signal recordings (group B); 0.73 for the group containing artifact-free, mild artifact, and moderate artifact signal recordings (group $\mathrm{C}$ ); and 0.68 for all signal recordings (group D). It can be seen that $P P R_{5 s}$ decreases as the level of artifacts increases.

As a comparison, we also calculated the segment-based evaluation metrics to evaluate the performance of our PGES detection method for classifying individual signal segments, including accuracy, recall $\left(R_{P G E S}\right)$, precision $\left(P_{P G E S}\right)$, and F1-score $\left(F 1_{P G E S}\right)$, as defined in Figure 7 , where $T P_{P G E S}$ is the number of segments detected as PGES and labeled as PGES by experts, $T P_{P G E S}$ is the number of segments detected as ISW and labeled as ISW by experts, Num PGES_expert $_{\text {is the total number }}$ of segments labeled as PGES by experts, Num $_{P G E S \_d e t e c t}$ is the total number of segments labeled as PGES by our detection method, and Num total $_{\text {is }}$ the total number of segments.

Table 1 shows the segment-based evaluation results of our method. The accuracy of each artifact group is over 0.92 . On the other hand, the recall, precision, and F1-score for each group are over $0.95,0.96$, and 0.95 , respectively.

Figure 7. The definitions of segment-based evaluation metrics.

$$
\begin{aligned}
& \text { Accuracy }=\frac{T P_{P G E S}+T N_{P G E S}}{N u m_{\text {total }}}(\mathrm{a}) \\
& \text { Recall }=\frac{T P_{P G E S}}{\text { Num } m_{P G E S \_ \text {expert }}}(\mathrm{b}) \\
& \text { Precision }=\frac{T P_{P G E S}}{\text { Num } m_{P G E S \_ \text {detect }}}(\mathrm{c}) \\
& F 1-\text { score }=2 \times \frac{\text { Recall } \times \text { Precision }}{\text { Recall }+ \text { Precision }}(\mathrm{d})
\end{aligned}
$$


Table 1. The traditional evaluation of postictal generalized electroencephalogram suppression (PGES) detection on each testing group.

\begin{tabular}{lllll}
\hline Evaluation metric & Group A $^{\mathrm{a}}$ & Group B & Group C $^{\mathrm{c}}$ & Group D $^{\mathrm{d}}$ \\
\hline Accuracy & 0.94 & 0.94 & 0.94 & 0.92 \\
Recall (R & 0.95 & 0.96 & 0.97 & 0.95 \\
Precision (PGES & 0.98 & 0.98 & 0.97 & 0.96 \\
F1-score (F1 & 0.97 & 0.96 & 0.97 & 0.95 \\
\hline
\end{tabular}

${ }^{\mathrm{a}}$ Only artifact-free signal recordings.

${ }^{\mathrm{b}}$ Artifact-free and mild artifact signal recordings.

${ }^{c}$ Artifact-free, mild artifact, and moderate artifact signal recordings.

${ }^{\mathrm{d}}$ All signal recordings.

\section{Discussion}

\section{Principal Findings}

We developed an automated PGES detection method based on EEG signals, which combined a random forest classifier and 3 correction rules. The main idea of our method was to leverage both signal features and the state transitions of brain activities. We evaluated the performance of our method using different artifact groups of signal recordings.

We reported both the segment-based evaluation results and the recording-based evaluation results. According to the segment-based evaluation results, our method achieved over 0.92 accuracy, 0.95 recall, 0.96 precision, and $0.95 \mathrm{~F} 1$-score for each artifact group. The results were consistent for each group, which indicates that our method performed well for classifying individual PGES signal segments (even for the group containing signal recording with severe artifacts).

However, as illustrated in Figure 6, the segment-based evaluation may not be able to demonstrate the actual PGES detection performance. In practical settings, the segment that was incorrectly detected as ISW would cause the wrong annotation of the PGES end time and result in an incorrect, significantly different PGES duration, which may mislead the risk assessment of SUDEP. Therefore, we introduced a way with direct practical relevance to evaluate automated PGES methods based on time distance, which is the time difference between the detected PGES period and the expert-annotated one.

On the basis of our recording and time distance-based evaluation, our PGES detection method achieved an average predicted time distance of 2.4 seconds and a $\mathrm{PPR}_{5 \mathrm{~s}}$ of 0.95 for artifact-free EEG signals. For signals with artifacts, the performance of this method varies according to the level of artifacts. For signals with mild artifacts, our method achieved a $P_{P R}$ s of 0.81 . However, as the number of signals with higher artifact levels (moderate) increased, the $\mathrm{PPR}_{5 \mathrm{~s}}$ dropped to 0.73 ; for signals with all artifact levels (artifact-free to severe), it dropped to 0.68 , and the average predicted time distance was 7.84 seconds. The artifact is the main challenge for PGES detection. To identify high-amplitude artifacts (severe level) that are not real brain activities, clinicians usually have to use different EEG patterns or even video recordings. In future work, we will focus on developing an approach for handling signals with high artifact levels (moderate and above). In particular, we plan to try dedicated artifact removal methods such as independent component analysis [46,47], regression analysis [48], and empirical method [49,50] to study whether such methods would help improving the performance of PGES detection.

Compared with the previous work [19], we used three additional types of features (time-domain features, wavelet-based features, and interchannel correlations) in the feature extraction step. For the classifier, we used random forest instead of boosting algorithms with logistic regression. We also introduced a new metric (predicted time distance) to evaluate an automated PGES detection method, and we reported evaluation results for both the segment-based method and our new metrics (no such evaluations were performed in the study by Theeranaew et al [19]).

\section{Conclusions}

We presented an automated method that combines the benefits of random forest classifier and correction rules for PGES detection using multichannel EEG recordings. Features from temporal, frequency, wavelet, and cross-coherence analyses provided valuable information to characterize PGES and ISW. Confidence-based rules were leveraged to correct sudden changes of PGES states. We introduced a new evaluation method for assessing PGES detection performance with more practical relevance. The evaluation results indicated that our method achieved a $\mathrm{PPR}_{5 \mathrm{~s}}$ of 0.95 for artifact-free EEG recordings. For EEG recordings with different artifact levels, the $\mathrm{PPR}_{5 \mathrm{~s}}$ varied from 0.68 to 0.81 . This study demonstrates that our combined random forest and rule-based approach can perform well in realistic settings for good quality EEG recordings.

\section{Acknowledgments}

This work was supported by the US National Institutes of Health (NIH) under grant U01NS090408. The content is solely the responsibility of the authors and does not necessarily represent the official views of the NIH. 


\section{Conflicts of Interest}

None declared.

\section{References}

1. Bentivoglio M, Cavalheiro EA, Kristensson K, Patel NB, editors. Neglected Tropical Diseases and Conditions of the Nervous System. Heidelberg: Springer; 2014.

2. Goldenberg MM. Overview of drugs used for epilepsy and seizures: etiology, diagnosis, and treatment. Pharm Ther 2010 Jul;35(7):392-415 [FREE Full text] [Medline: 20689626]

3. Good DC. Chapter 51. Episodic neurologic symptoms. In: Walker HK, Hall WD, Hurst JW, editors. Clinical Methods: The History, Physical and Laboratory Examinations. Third Edition. Boston: Butterworths; 1990.

4. Lhatoo SD, Nei M, Raghavan M, Sperling M, Zonjy B, Lacuey N, et al. Nonseizure SUDEP: Sudden unexpected death in epilepsy without preceding epileptic seizures. Epilepsia 2016 Jul;57(7):1161-1168 [FREE Full text] [doi: 10.1111/epi.13419] [Medline: 27221596]

5. Thurman DJ, Hesdorffer DC, French JA. Sudden unexpected death in epilepsy: assessing the public health burden. Epilepsia 2014 Oct;55(10):1479-1485 [FREE Full text] [doi: 10.1111/epi.12666] [Medline: 24903551]

6. Maguire M, Jackson C, Marson A, Nolan SJ. Treatments for the prevention of sudden unexpected death in epilepsy (SUDEP). Cochrane Database Syst Rev 2016 Jul 19;7:CD011792 [FREE Full text] [doi: 10.1002/14651858.CD011792.pub2] [Medline: 27434597]

7. Sperling MR. Sudden unexplained death in epilepsy. Epilepsy Curr $2001 \mathrm{Sep} ; 1(1): 21-23$ [FREE Full text] [doi: 10.1046/j.1535-7597.2001.00012.x] [Medline: 15309034]

8. Bertram EH. Electrophysiology in epilepsy surgery: roles and limitations. Ann Indian Acad Neurol 2014 Mar;17(Suppl 1):S40-S44 [FREE Full text] [doi: 10.4103/0972-2327.128649] [Medline: 24791088]

9. Worrell G, Gotman J. High-frequency oscillations and other electrophysiological biomarkers of epilepsy: clinical studies. Biomark Med 2011 Oct;5(5):557-566 [FREE Full text] [doi: 10.2217/bmm.11.74] [Medline: 22003904]

10. Smith SJ. EEG in the diagnosis, classification, and management of patients with epilepsy. J Neurol Neurosurg Psychiatry 2005 Jun;76(Suppl 2):ii2-ii7 [FREE Full text] [doi: 10.1136/jnnp.2005.069245] [Medline: 15961864]

11. Staba RJ, Stead M, Worrell GA. Electrophysiological biomarkers of epilepsy. Neurotherapeutics 2014 Apr;11(2):334-346 [FREE Full text] [doi: 10.1007/s13311-014-0259-0] [Medline: 24519238]

12. Vilella L, Lacuey N, Hampson JP, Rani MR, Loparo K, Sainju RK, et al. Incidence, recurrence, and risk factors for peri-ictal central apnea and sudden unexpected death in epilepsy. Front Neurol 2019;10:166 [FREE Full text] [doi: 10.3389/fneur.2019.00166] [Medline: 30890997]

13. Wu S, Issa NP, Rose SL, Ali A, Tao JX. Impact of periictal nurse interventions on postictal generalized EEG suppression in generalized convulsive seizures. Epilepsy Behav 2016 May;58:22-25. [doi: 10.1016/j.yebeh.2016.02.025] [Medline: 26994879]

14. Lhatoo SD, Faulkner HJ, Dembny K, Trippick K, Johnson C, Bird JM. An electroclinical case-control study of sudden unexpected death in epilepsy. Ann Neurol 2010 Dec;68(6):787-796. [doi: 10.1002/ana.22101] [Medline: 20882604]

15. Esmaeili B, Kaffashi F, Theeranaew W, Dabir A, Lhatoo SD, Loparo KA. Post-ictal modulation of baroreflex sensitivity in patients with intractable epilepsy. Front Neurol 2018;9:793 [FREE Full text] [doi: 10.3389/fneur.2018.00793] [Medline: 30319527]

16. Alexandre V, Mercedes B, Valton L, Maillard L, Bartolomei F, Szurhaj W, REPO2MSE study group. Risk factors of postictal generalized EEG suppression in generalized convulsive seizures. Neurology 2015 Nov 3;85(18):1598-1603. [doi: 10.1212/WNL.0000000000001949] [Medline: 26333799]

17. Kuo J, Zhao W, Li C, Kennedy JD, Seyal M. Postictal immobility and generalized EEG suppression are associated with the severity of respiratory dysfunction. Epilepsia 2016 Mar;57(3):412-417 [FREE Full text] [doi: 10.1111/epi.13312] [Medline: 26763069]

18. Asadollahi M, Noorbakhsh M, Simani L, Ramezani M, Gharagozli K. Two predictors of postictal generalized EEG suppression: tonic phase duration and postictal immobility period. Seizure 2018 Oct;61:135-138. [doi: 10.1016/j.seizure.2018.08.009] [Medline: 30142618]

19. Theeranaew W, McDonald J, Zonjy B, Kaffashi F, Moseley BD, Friedman D, et al. Automated detection of postictal generalized EEG suppression. IEEE Trans Biomed Eng 2018 Feb;65(2):371-377 [FREE Full text] [doi: 10.1109/TBME.2017.2771468] [Medline: 29346105]

20. Baumgartner C, Koren JP, Rothmayer M. Automatic computer-based detection of epileptic seizures. Front Neurol 2018;9:639 [FREE Full text] [doi: 10.3389/fneur.2018.00639] [Medline: 30140254]

21. Bhattacharyya A, Pachori RB. A multivariate approach for patient-specific EEG seizure detection using empirical wavelet transform. IEEE Trans Biomed Eng 2017;64(9):2003-2015. [doi: 10.1109/tbme.2017.2650259]

22. Zahra A, Kanwal N, Rehman NU, Ehsan S, McDonald-Maier KD. Seizure detection from EEG signals using Multivariate Empirical Mode Decomposition. Comput Biol Med 2017 Sep 1;88:132-141. [doi: 10.1016/j.compbiomed.2017.07.010] [Medline: 28719805] 
23. Fergus P, Hussain A, Hignett D, Al-Jumeily D, Abdel-Aziz K, Hamdan H. A machine learning system for automated whole-brain seizure detection. Appl Comput Inform 2016;12(1):70-89. [doi: 10.1016/j.aci.2015.01.001]

24. Pisarchik AN, Grubov VV, Maksimenko VA, Lüttjohann A, Frolov NS, Marqués-Pascual C, et al. Extreme events in epileptic EEG of rodents after ischemic stroke. Eur Phys J Spec Top 2018;227(7-9):921-932. [doi: 10.1140/epjst/e2018-800019-1]

25. Frolov NS, Grubov VV, Maksimenko VA, Lüttjohann A, Makarov VV, Pavlov AN, et al. Statistical properties and predictability of extreme epileptic events. Sci Rep 2019 May 10;9(1):7243 [FREE Full text] [doi: 10.1038/s41598-019-43619-3] [Medline: 31076609]

26. Lhatoo S, Noebels J, Whittemore V, NINDS Center for SUDEP Research. Sudden unexpected death in epilepsy: identifying risk and preventing mortality. Epilepsia 2015 Nov;56(11):1700-1706 [FREE Full text] [doi: 10.1111/epi.13134] [Medline: 26494436]

27. Jobson JD. Applied Multivariate Data Analysis: Volume II: Categorical and Multivariate Methods. New York: Springer-Verlag; 2012.

28. Charbonnier S, Zoubek L, Lesecq S, Chapotot F. Self-evaluated automatic classifier as a decision-support tool for sleep/wake staging. Comput Biol Med 2011 Jun;41(6):380-389. [doi: 10.1016/j.compbiomed.2011.04.001] [Medline: 21497802]

29. Hjorth B. EEG analysis based on time domain properties. Electroencephalogr Clin Neurophysiol 1970 Sep;29(3):306-310. [doi: 10.1016/0013-4694(70)90143-4] [Medline: 4195653]

30. Redmond S, Heneghan C. Cardiorespiratory-based sleep staging in subjects with obstructive sleep apnea. IEEE Trans Biomed Eng 2006 Mar;53(3):485-496. [doi: 10.1109/TBME.2005.869773] [Medline: 16532775]

31. Oh SH, Lee YR, Kim HN. A novel EEG feature extraction method using Hjorth parameter. Int J Electron Electr Eng 2014;2(2):106-110. [doi: 10.12720/ijeee.2.2.106-110]

32. Mallat S. A Wavelet Tour of Signal Processing. Amsterdam, Netherlands: Elsevier; 1999.

33. Misiti M, Misiti Y, Oppenheim G, Poggi JM. Luleå tekniska universitet, LTU. 1996. Wavelet Toolbox 4: User's Guide URL: https://www.ltu.se/cms fs/1.51590!/wavelet\%20toolbox\%204\%20user's\%20guide\%20(larger\%20selection).pdf [accessed 2020-01-27]

34. Gysels E, Celka P. Phase synchronization for the recognition of mental tasks in a brain-computer interface. IEEE Trans Neural Syst Rehabil Eng 2004;12(4):406-415. [doi: 10.1109/tnsre.2004.838443]

35. Gouy-Pailler C, Achard S, Rivet B, Jutten C, Maby E, Souloumiac A, et al. Topographical Dynamics of Brain Connections for the Design of Asynchronous Brain-Computer Interfaces. In: Proceedings of the 2007 29th Annual International Conference of the IEEE Engineering in Medicine and Biology Society. 2007 Presented at: IEMBS'07; August 22-26, 2007; Lyon, France p. 2520-2523. [doi: 10.1109/iembs.2007.4352841]

36. Grosse-Wentrup M. Understanding Brain Connectivity Patterns during Motor Imagery for Brain-Computer Interfacing. In: Proceedings of the 2008 Conference on Neural Information Processing Systems. 2008 Presented at: NIPS'08; December 8-11, 2008; Vancouver, British Columbia, Canada URL: https://papers.nips.cc/paper/ 3505-understanding-brain-connectivity-patterns-during-motor-imagery-for-brain-computer-interfacing.pdf

37. Wei Q, Wang Y, Gao X, Gao S. Amplitude and phase coupling measures for feature extraction in an EEG-based brain-computer interface. J Neural Eng 2007 Jun;4(2):120-129. [doi: 10.1088/1741-2560/4/2/012] [Medline: 17409486]

38. Chung YG, Kim MK, Kim SP. Inter-channel Connectivity of Motor Imagery EEG Signals for a Noninvasive BCI Application. In: Proceedings of the 2011 International Workshop on Pattern Recognition in NeuroImaging.: IEEE; 2011 Presented at: PRNI'11; May 16-18, 2011; Seoul, South Korea. [doi: 10.1109/prni.2011.9]

39. Díaz MH, Córdova FM, Cañete L, Palominos F, Cifuentes F, Rivas G. Inter-channel correlation in the EEG activity during a cognitive problem solving task with an increasing difficulty questions progression. Procedia Comput Sci 2015;55:1420-1425. [doi: 10.1016/j.procs.2015.07.136]

40. Fraiwan L, Lweesy K, Khasawneh N, Wenz H, Dickhaus H. Automated sleep stage identification system based on time-frequency analysis of a single EEG channel and random forest classifier. Comput Methods Programs Biomed 2012 Oct;108(1):10-19. [doi: 10.1016/j.cmpb.2011.11.005] [Medline: 22178068]

41. Li X, Cui L, Tao S, Chen J, Zhang X, Zhang G. HyCLASSS: a hybrid classifier for automatic sleep stage scoring. IEEE J Biomed Health Inform 2018 Mar;22(2):375-385. [doi: 10.1109/JBHI.2017.2668993] [Medline: 28222004]

42. Koley B, Dey D. An ensemble system for automatic sleep stage classification using single channel EEG signal. Comput Biol Med 2012 Dec;42(12):1186-1195. [doi: 10.1016/j.compbiomed.2012.09.012] [Medline: 23102750]

43. Breiman L. Random forests. Mach Learn 2001;45(1):5-32 [FREE Full text] [doi: 10.1023/A:1010933404324]

44. Längkvist M, Karlsson L, Loutfi A. Sleep Stage Classification Using Unsupervised Feature Learning. Adv Artif Neural Syst 2012;2012:1-9. [doi: 10.1155/2012/107046]

45. Rodríguez-Sotelo J, Osorio-Forero A, Jiménez-Rodríguez A, Cuesta-Frau D, Cirugeda-Roldán E, Peluffo D. Automatic sleep stages classification using EEG entropy features and unsupervised pattern analysis techniques. Entropy 2014;16(12):6573-6589. [doi: 10.3390/e16126573]

46. Makeig S, Bell AJ, Jung TP, Sejnowski TJ. Independent component analysis of electroencephalographic data. Adv Neural Inf Process Syst 1996;8(8):145-151 [FREE Full text] 
47. Pavlov AN, Hramov AE, Koronovskii AA, Sitnikova EY, Makarov VA, Ovchinnikov AA. Wavelet analysis in neurodynamics. Phys-Usp 2012;55(9):845-875. [doi: 10.3367/ufne.0182.201209a.0905]

48. Gratton G, Coles MG, Donchin E. A new method for off-line removal of ocular artifact. Electroencephalogr Clin Neurophysiol 1983;55(4):468-484. [doi: 10.1016/0013-4694(83)90135-9]

49. Grubov VV, Runnova AE, Koronovskii AA, Hramov AE. Adaptive filtering of electroencephalogram signals using the empirical-modes method. Tech Phys Lett 2017;43(7):619-622. [doi: 10.1134/s1063785017070070]

50. Grubov VV, Sitnikova E, Pavlov AN, Koronovskii AA, Hramov AE. Recognizing of stereotypic patterns in epileptic EEG using empirical modes and wavelets. Physica A 2017 Nov;486:206-217. [doi: 10.1016/j.physa.2017.05.091]

\author{
Abbreviations \\ AE: amplitude energy \\ CSR: Center for Sudden Unexpected Death in Epilepsy Research \\ EEG: electroencephalogram \\ EMU: epilepsy monitoring unit \\ GTCS: generalized tonic-clonic seizures \\ ISW: intermittent slow wave \\ NIH: National Institutes of Health \\ PGES: postictal generalized electroencephalogram suppression \\ $\mathbf{P P R}_{5 \mathrm{~s}}$ : 5-second tolerance-based positive prediction rate \\ SUDEP: sudden unexpected death in epilepsy
}

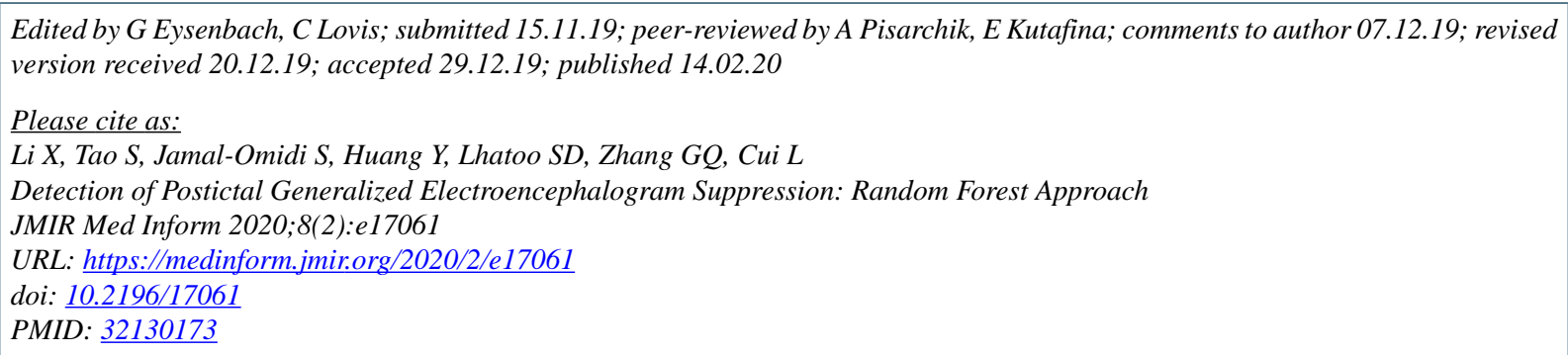

(CXiaojin Li, Shiqiang Tao, Shirin Jamal-Omidi, Yan Huang, Samden D Lhatoo, Guo-Qiang Zhang, Licong Cui. Originally published in JMIR Medical Informatics (http://medinform.jmir.org), 14.02.2020. This is an open-access article distributed under the terms of the Creative Commons Attribution License (https://creativecommons.org/licenses/by/4.0/), which permits unrestricted use, distribution, and reproduction in any medium, provided the original work, first published in JMIR Medical Informatics, is properly cited. The complete bibliographic information, a link to the original publication on http://medinform.jmir.org/, as well as this copyright and license information must be included. 